\section{PTH-66 CORRELATION OF PEESS AND EREFS SCORES WITH HISTOLOGICAL ACTIVITY IN CHILDREN WITH EOSINOPHILIC OESOPHAGITIS (EOE)}

Vaia Zouzo*, Adepoju Akinlolu, Alexandra Patrick, Emma Jones, William Simmons, Rajeev Shukla, Marcus KH Auth. Alder Hey Childrens' Hospital, Liverpool, UK

\subsection{6/gutjnl-2021-BSG.245}

Introduction Management of children with EoE is hampered by lack of reliable biomarkers. Although the Paediatric Eosinophilic Esophagitis Symptom Score (PEESS v2.0) has been validated as a marker for clinical activity in children and the Endoscopic Reference Score (EREFS) in adults, their use for clinical application has not been evaluated in children in the UK.

Aims of this pilot study were:

- Correlation of clinical activity (PEESS) with endoscopic grading (EREFS) and standard oesophageal histology

(i) Feasibility in clinical practice.

Methods Retrospective review of a single consultant's cohort of children with $\operatorname{EoE}(n=13)$ over 18 months $(2019-20)$ providing $\mathrm{n}=19$ assessments.

PEESSv2.0 comprises 20 questions about symptom frequency and severity in domains dysphagia, reflux, nausea/vomiting, and pain generating scores from 0-4. EREFS depicts endoscopic EoE features (oedema, rings, furrowing, exudate, stricture) into a grading score of severity. Pearson's correlation test was used to indicate significance $(\mathrm{p}<0.05)$.

Results $84.6 \%$ of children were male with a mean age of 13.7 years $(8.7-17.4 \mathrm{yr})$.

In $47.3 \%(9 / 19)$ histologically active EoE $[\geq 15$ eosinophils per high power field (eos/hpf)] was found. Mean peak eos/hpf were $4.7(0-35)$ in proximal, $14.9(0-100)$ in mid and 20.8 $(0-80)$ in distal oesophagus.

Mean PEESS score was 20 (mild) and IQR 0-31.5 (range $0-68$ ), indicating mild to moderate activity. There was positive correlation of PEESS scores with the density of eosinophils in the mid $(p=0.004)$ and distal $(p=0.0009)$ oesophagus, but not in proximal $(\mathrm{p}=0.23)$.

Mean EREFS score was 1.4 (range 0-3), with furrowing and micro abscess being the most common findings. The correlation of EREFS with PEESS was not significant $(\mathrm{p}=0.43)$.

Treatment at time of assessment consisted in 63\% (12/19) of children of oral viscous budesonide, $21 \%$ (4/19) of PPI, $11 \%(2 / 19)$ of elimination diet (ED), and 5\% (1/19) of combination therapy.

Conclusion In our pilot study, routine use of PEESS in clinical settings was practical, feasible, and helped to evaluate more objectively efficacy of treatment.

Similar to a study in the US, we confirmed a correlation of histologic and clinical activity (PEESS) of EoE in children in the UK. Endoscopic EREFS scores may be helpful to assess longitudinally disease activity and mucosal alternations in patients with EoE.

If applied consistently, these scores can be easily applied to monitor disease activity, provide guidance on timing and frequency of endoscopic reassessment, indicate need for treatment escalation, and may help to recognise earlier beginning signs of complications.

\section{PTH-67 LOW RATE OF POST-FUNDOPLICATION DYSPHAGIA IN PATIENTS WITH PRE-OPERATIVE OESOPHAGEAL APERISTALSIS}

Raphael Luber*, Terry Wong, Radu Rusu, Ismail Miah, Guiping Sui, Abrie Botha, Andrew Davies, James Gossage, Jason Dunn, Jafar Jafari, Sebastian Zeki. Guy's and St Thomas' Hospitals, London, UK

\subsection{6/gutjnl-2021-BSG.246}

Introduction Limited data exist on the safety of fundoplication surgery in patients with oesophageal aperistalsis, with some considering it a contraindication due to risk of dysphagia. We aimed to assess rates \& predictors of dysphagia in such patients who underwent fundoplication.

Methods Patients with oesophageal aperistalsis based on the Chicago classification v3.0 who underwent fundoplication for confirmed symptomatic reflux were identified at a single centre. Pre-operative high-resolution manometries (HRM) were reviewed, with standard parameters as well as presence of contractile reserve (peristalsis with distal contractile integral (DCI) >100) following multiple water swallows (MWS), solids, or saliva recorded. Patients were excluded if they had an alternate manometric diagnosis, incomplete HRM, or antireflux surgery pre-HRM. The hospital odynophagia \& dysphagia questionnaire (HODQ) was performed post-operatively. HODQ $>6$ was considered abnormal.

Results 17 patients (7 male, median [IQR] age at surgery 53y [38.5-66.5]) with a median time to post-operative scoring of 34 (18-52) months were included; 13 (76\%) had a normal post-operative HODQ. Complete fundoplication was performed in 12 patients, of which $2(17 \%)$ had an abnormal HODQ. There was no significant difference in demographics, type of surgery, or pre-operative HRM parameters between

Abstract PTRH-67 Table 1 Clinical \& HRM characteristics

\begin{tabular}{|c|c|c|c|}
\hline & $\begin{array}{l}\text { Normal post op } \\
\text { HODQ, } n=13\end{array}$ & $\begin{array}{l}\text { Abnormal post op } \\
\text { HODQ, } n=4\end{array}$ & $\mathrm{p}$ \\
\hline Male, n (\%) & $4(30.8)$ & $3(75)$ & 0.25 \\
\hline $\begin{array}{l}\text { Age (years) at operation, median } \\
\text { (IQR) }\end{array}$ & $59(38.5-69)$ & $47(32.8-62.8)$ & 0.36 \\
\hline $\begin{array}{l}\text { Pre-op 24hr acid exposure time } \\
\text { (mins), median (IQR) }\end{array}$ & $150(122-178.2)$ & $112.5(52.3-173.1)$ & 0.57 \\
\hline Pre-op dysphagia, n (\%) & $2(15.4)$ & $2(50)$ & 0.22 \\
\hline Complete fundoplication, n (\%) & $10(76.9)$ & $2(50)$ & 0.54 \\
\hline Hernia, $\mathrm{n}(\%)$ & $6(46.2)$ & $2(50)$ & 1.0 \\
\hline $\begin{array}{l}\text { Integrated relaxation pressure, } \\
\text { median (IQR) }\end{array}$ & $2.6(1.2-4.7)$ & $0.75(-0.6-5.6)$ & 0.57 \\
\hline MWS contractile reserve, $\mathrm{n}(\%)$ & $1(7.7)$ & $1(25)$ & 0.57 \\
\hline $\begin{array}{l}\text { Solid swallow contractile reserve, } n \\
\text { (\%) }\end{array}$ & $8(61.5)$ & $3(75)$ & 0.81 \\
\hline $\begin{array}{l}\text { Random saliva swallow contractile } \\
\text { reserve, } n(\%)\end{array}$ & $7(53.8)$ & $1(25)$ & 0.58 \\
\hline Solids, DCI max, median (IQR) & $491.1(0-1185.7)$ & $668.3(161.2-1052.8)$ & 0.71 \\
\hline Saliva, DCI max, median (IQR) & $272.7(0-415.5)$ & $0(0-358.4)$ & 0.62 \\
\hline $\begin{array}{l}\text { Total swallows with } \mathrm{DCl}>100 \text {, } \\
\text { median (IQR) }\end{array}$ & $3(0-7)$ & $5(1-6)$ & 0.82 \\
\hline
\end{tabular}


those with a normal vs. abnormal post-operative HODQ (Table 1). Of those with an abnormal post-operative HODQ, $50 \%$ had pre-operative dysphagia \& all were managed conservatively. There was no correlation between HODQ \& maximum DCI achieved with solid or saliva swallows, nor overall number of swallows with DCI $>100$.

Conclusion The majority (76\%) of patients with oesophageal aperistalsis did not have dysphagia post-operatively. There were no clinical or HRM predictors of dysphagia, however this was limited by the small numbers who had post-operative dysphagia.

\section{PTH-68 SCHATZKI RINGS ARE COMMONLY MISDIAGNOSED IN PATIENTS WITH EOSINOPHILIC OESOPHAGITIS}

Sebastian Zeki*, Terry Wong, Jason Dunn. Guy's and St Thomas' NHS Trust, London, UK

\subsection{6/gutjnl-2021-BSG.247}

Introduction Eosinophilic oesophagitis (EoE) is an increasingly common disorder characterised by the accumulation of eosinophils within the oesophageal epithelium. Schatzki rings are defined as thin mucosal fibrotic rings most commonly seen at the GOJ. Schatzki rings can be mistaken for fibrotic strictures of EoE and it is therefore important to biopsy all rings and strictures to ascertain whether eosinophils are present. The aim of the current study was to assess what proportion of patients undergoing diagnostic gastroscopy where a Schatzki ring is identified, in fact have EoE when biopsied.

Methods All endoscopic and pathology text extraction was performed using EndoMineR. All free text endoscopy reports containing the term 'Schatzki' and any spelling variants were identified between 1st Jan 2007 and 23rd January 2021. Typical EoE endoscopic findings were extracted (Table 1). The histopathology free text from any oesophageal biopsies from the same cohort was analysed and results showing the presence of $>15$ eosinophils per high power field were identified as diagnostic of EoE.

In addition the endoscopies from all patients with EoE diagnosed at any point using for the same time period on biopsy from the oesophagus were obtained from the pathology database and manually validated. EoE patients with strictures or rings were identified as being fibrostenotic (EoE-FS) To determine whether patients with EoE and Schatzki rings (EoESchatzki) were likely to be the same phenotype as EoE-FS at

\begin{tabular}{|c|c|c|c|}
\hline Characteristic & EoE-FS, $N=67$ & EoE-Schatzki, $N=35$ & $p$-value ${ }^{2}$ \\
\hline Sex & & & 0.5 \\
\hline$F$ & $14(21 \%)$ & $10(29 \%)$ & \\
\hline$M$ & $53(79 \%)$ & $25(71 \%)$ & \\
\hline Age at diagnosis (yrs) & $41(14)$ & $51(16)$ & 0.005 \\
\hline Atopic history & $26(39 \%)$ & $9(26 \%)$ & 0.2 \\
\hline Hiatus Hernia & $10(15 \%)$ & $20(57 \%)$ & $<0.001$ \\
\hline Furrow & $39(60 \%)$ & $7(20 \%)$ & $<0.001$ \\
\hline Oedema & $20(30 \%)$ & $1(2.9 \%)$ & 0.003 \\
\hline Tracheal & $38(73 \%)$ & $31(97 \%)$ & 0.007 \\
\hline WhiteSpots & $21(31 \%)$ & $2(5.7 \%)$ & 0.007 \\
\hline
\end{tabular}

endoscopy were then compared to those the EoE and no Schatzki rings for all the variables detailed above

Results 124 endoscopies where a Schatzki ring was described, were identified (average age at diagnosis: 52 (15) years, M:F 78 (63\%): 46 (37\%)). Of these, 35 (28\%) endoscopies showed eosinophil count of $>15$ eosinophils/hpf. EoE-Schatzki patients were more likely than EoE-FS to have a hiatus hernia (20 (57\%) vs $10(15 \%))$, trachealization (31 (97\%) $38(73 \%))$ and have a higher mean age group (51 yrs (16)) vs 41 yrs (14)) and less likely to have inflammatory findings of furrows (7 (20\%) vs $39(60 \%))$, oedema $(1(2.9 \%)$ vs $20(30 \%))$ and white exudates $(2(5.7 \%)$ vs $21(31 \%))$.

Conclusions Schatzki rings are commonly diagnosed in patients with EoE and should be biopsied to confirm the presence of EoE. Further work is necessary to determine if the Schatzki rign may represent a novel EoE phenotype which is different to, or a variant of fibrostenotic disease.

\section{PTH-69 SHORT SEGMENT NON DYSPLASTIC BARRETT'S IS OFTEN SURVEYED TOO EARLY BY NON-EXPERTS}

Sebastian Zeki*, Jason Dunn. Guy's And St Thomas' NHS Trust, London, UK

\subsection{6/gutjnl-2021-BSG.248}

Introduction Dedicated Barrett's oesophagus (BO) surveillance endoscopy lists improve dysplasia detection. However, the decision when to endoscopically survey a patient is often done by non-experts. Since 2014, the guidelines regarding surveillance timing have changed and guidance is now based on the length of the maximum extent of the Barrett's segment as well as the presence or absence of IM in BO less than $3 \mathrm{~cm}$ in maximal extent. It has been shown that adherence to guidance on surveillance timing is poorly performed in the US. We sought to assess the adherence in a single centre in the UK with a high throughput of Barrett's cases as well as determine whether the timing of surveillance endoscopies was best performed by experts or non-expert endoscopists.

Methods All endoscopies performed where BO was detected and biopsied were identified between January 1st 2014 and January 10th 2021. Only patients who had undergone more than one surveillance endoscopy and in whom dysplasia or cancer had never been detected in the specified time frame were analysed. The endoscopy specific natural language pipeline EndoMineR extracted the pathological stage and Prague score for each endoscopy. The difference between the date of the suggested guideline-based follow up endoscopy and the actual date was calculated. An endoscopy was labelled as being too early or late if performed more or less than 6 months prior to the limit of the suggested date range respectively.

Results Since 2014109 follow up endoscopies in 92 patients where the index endoscopy for non-dysplastic Barrett's oesophagus was also performed since 2014 were identified (average age 65 (14) years, M:F 87 (80\%) : 22 (20\%), average C Stage $2.9 \mathrm{~cm}$ (3.4) average $M$ stage $4.0 \mathrm{~cm}$ (3.6). Of these endoscopies, 22 were classified as $<3 \mathrm{~cm}$ in maximum length with no IM (Rule 1), 28 as $<3 \mathrm{~cm}$ in maximum length with IM (Rule 2) and 59 as $>3 \mathrm{~cm}$ length (Rule 3). The majority $35(70 \%)$ of short segment, non dysplastic BO was being performed inappropriately early with endoscopy occurring a mean of 653.71 (265) days early for these groupings. The decision for further surveillance for the Rule 3 group was more accurate with 21 (35.59\%) performed too early. The 